F. Child Lang. 24 ( 1997 ), 5 I I-533. Printed in the United Kingdom

(C) 1997 Cambridge University Press

\title{
Linguistic cues in the acquisition of number words*
}

\author{
PAUL BLOOM AND KAREN WYNN \\ University of Arizona \\ (Received 7 Fuly 1995. Revised 3 February 1997)
}

\begin{abstract}
Previous research has shown that children go through a stage in which they know that the number words each refer to a distinct numerosity, yet do not know WHICH numerosity each number word picks out (Wynn, I 992). How do children attain this level of knowledge? We explore the possibility that particular properties of how number words are used within sentences inform children of the semantic class to which they belong. An analysis of transcripts of the spontaneous speech of three one- and two-year-old children and their parents (from the CHILDES database; MacWhinney \& Snow, I 990) suggests that the relevant cues are available as input in parents' speech to children, and that children generally honour these properties of number words in their own speech. Implications of this proposal for word learning more generally are discussed.
\end{abstract}

\section{INTRODUCTION}

Long before they start to learn words, infants have a rich understanding of number. Seven-month-olds who are repeatedly presented with pictures containing three items will show increased interest when shown a new picture with two items, and vice-versa (Starkey \& Cooper, I 980). Similarly, six-month-olds can distinguish two jumps of a puppet from three jumps of a puppet (Wynn, I 996) and two sounds from three sounds (Starkey, Spelke \& Gelman, I 990). Finally, if five-month-olds are shown one object added to another identical object, or one object removed from a collection of two identical objects, they will look longer, indicating surprise, when the number of objects revealed as the result of this operation is numerically incorrect than when the result is correct, suggesting that they possess some rudimentary appreciation of addition and subtraction (Wynn, I992a).

[*] We thank three anonymous reviewers for helpful commments on an earlier draft and Karen Kemtes for her very able assistance on a preliminary set of analyses. The first author was supported by grants from the Spencer Foundation and from the Sloan Foundation. The second author was supported by an NICHD FIRST Award (Grant no. I R29 HD29857). Address for correspondence: Paul Bloom/Karen Wynn, Department of Psychology, University of Arizona, Tucson, AZ 85721, USA. email: bloom (a)u.arizona.edu orwynn@ccit.arizona.edu. 
These findings suggest that children's task in learning small number words - such as the English words two and three - is to map them onto concepts already present. Just how children establish this mapping, however, is an interesting puzzle. The word two in the phrase two black cats does not describe any individual in the external world, nor does it refer to a property that any individual in the world might possess. In this regard, it differs from the noun cats, which is understood as describing cats, or the adjective black, which is understood as describing a property that each of the individual cats might have. Rather two is a predicate that applies to the set of cats. More generally, as Frege (I893/ı980) has argued, numbers are predicates of sets of individuals.

This raises certain problems from the standpoint of a theory of language acquisition. For one thing, sets are notoriously abstract entities. One can see and hear cats, but nobody has ever been wakened in the middle of the night by the yowling of a set. The apprehension of sets most likely requires some cognitive capacity above and beyond the normal apprehension of entities in the world (Maddy, I990). For another, children often experience number words as they are used within the routine of linguistic counting, in which each number word is assigned to an individual item in a one-to-one correspondence (Gelman \& Gallistel, I 978). Given children's disposition to take novel words as referring to individuals (Macnamara, I982; Markman, I 989), it would seem especially difficult for them to learn that the number words do NOT refer to the individual items that are being counted, or even to properties of these items, but rather to properties of sets of these items.

When do children come to learn the number words, and what is their initial understanding of what these words mean? This issue was addressed in a longitudinal study (Wynn, I992 $b$ ). The logic of the study required that children know the precise meaning of the word one - that it applies to only a single item, and not to several items. Two-and-a-half to three-and-a-halfyear-olds were tested for this knowledge, and those who passed this pretest (almost all did so) were then presented with a series of tasks over a sevenmonth period. In the task most relevant here, children were shown pairs of pictures. In each pair, one picture depicted a single item of a given kind of object, and the other depicted several (between two and six) items of the same kind but of a different colour. For each pair, children were asked to identify the picture that showed a particular number of objects, e.g. 'Can you show me the four fish/Can you show me the one fish?' (the items' names always had the same plural as singular forms, so as not to provide morphological cues to numerosity). They were also asked questions with nonsense words, e.g. 'Can you show me the zoop fish?'

The reasoning was that if children know that a number word - other than one - refers to a specific numerosity, they should infer that it does not refer to the same numerosity as the word one. In the questions contrasting one 
object with multiple objects, then, they should choose the correct picture by a process of elimination. For instance, imagine children shown a picture of one fish and a picture of four fish and asked to point to the four fish. If they know that four refers to a numerosity, and also know that different number words within a language must refer to different numerosities (in accord with the principle of contrast; Clark, I988) then, even if they do not know the precise meaning of four, they should point to the four fish. In contrast, if they do not know that a given number word picks out a specific numerosity, they will have no basis for contrasting it with one, and should respond as they do when asked a question with a nonsense word, such as 'Can you show me the zoop fish ?' - they should be equally likely to point to either picture.

To test if children knew WHICH numerosity a given word picks out, they were shown pairs of pictures, one containing the number of items corresponding to the word in question, the other containing that number plus one. For example, to test whether a child knows the precise meaning of the word four, she would be shown a picture of four fish and one of five fish, and would be asked 'Can you show me the four fish?' on some trials and 'Can you show me the five fish?' on others.

Even the youngest children (two-and-a-half-year-olds) succeeded when one of the pictures in the pair contained a single item. They correctly pointed out the number asked for $94 \%$ of the time. They thus showed an understanding that the number words pick out numerosities. However, despite this early knowledge, it took children nearly a full additional year to learn which words refer to which numerosities. For instance, a two-and-a-half-year-old who knows that four is a number word, as shown by the fact that she would never point to the single fish when asked 'Can you show me the four fish', might go for a year not knowing that four refers to four and not to five (i.e. when presented with a picture of four fish and a picture of five fish, the child will treat the question 'Can you show me the four fish?' in the same random fashion as she treats 'Can you show me the zoop fish?').

These results suggest that children go through a lengthy developmental stage in which they know that words like two and three refer to numerosities, but do not know which numerosities. This phenomenon is difficult to explain under the traditional empiricist view of how number words are learned (e.g. Mill, r 843/r 973). Under this view, children see a set of objects, perceive their numerosity (e.g. twoness), hear a word used to refer to the set (e.g. 'two'), and, over the course of repeated pairings of this type, come to learn the meaning of the number word. Such an account has obvious limitations. At best, it could only work for small numbers: we might perceive twoness without conscious counting but we surely do not perceive fourteenness or one-hundred-and-eightness in this manner; the names for these numerosities need to be acquired in some other way. But even for small numbers, it cannot explain the developmental sequence discussed above. It 
posits that children directly map words onto the perceptions that arise when exposed to different sets of objects and thus fails to account for a lengthy stage in which children can clearly DISTINGUISH between two and three entities (recall that even young infants can do this), know that two and three are number words - but do not yet know precisely what two and three mean.

A more promising theory involves children's ability to count. Gelman and her colleagues (e.g. Gelman \& Gallistel, r 978; Gelman, Meck \& Merkin, I 986) have suggested that children possess an innate set of counting principles that underlie their knowledge of counting, and they have found that children are highly competent at counting at a young age. They further posit that children learn the meanings of the number words by attending to how they are used in the counting routine.

There is evidence, however, against this account. First, children seem to achieve their initial understanding that the number words refer to numerosities (without knowing the precise numerosities that they refer to) long before they understand that counting determines the numerosity of a set that is, before they realize that the counting routine has anything to do with number (e.g. Fuson, I988; Wynn, I990). Secondly, if children did learn number words through mapping them onto the ordered counting sequence, one would expect them to understand, very early on, that, e.g. the word three can only be applied to sets of three, and that it picks out larger sets than the word two and smaller sets than the word four. They should know this by having learned that three is the third word in the English counting series, and that it follows two and precedes four - knowledge that is present very early. The fact that children go for a sustained period without this understanding is thus a puzzle under this account.

A different hypothesis, which we explore in this paper, is that linguistic cues may play a significant role in children's acquisition of number word meaning. Words are not typically used in isolation, but usually occur amongst other words, within sentences. Information provided by how a word is used within a sentence can be a rich source of knowledge about its meaning. There are two general kinds of linguistic cues that exist.

One type of information is syntactic. Across all languages, there are rich correspondences between syntax and semantics, and thus the syntactic context within which a word occurs will often reflect certain aspects of its meaning. This possibility of syntactic cues as a route to word meaning acquisition was first raised by Brown ( I 957), who found that preschoolers are sensitive to whether a word is a verb, a count noun, or a mass noun when determining whether it denotes an event, an object, or a substance. This finding was extended by Katz, Baker \& Macnamara (1974), who found that children younger than two are sensitive to the presence or absence of a determiner ('This is a zav'vs. 'This is zav') when determining whether a novel word is a name for a kind or a proper name. More recently, children's 
sensitivity to syntax-semantics mappings has been explored in the area of verb learning (e.g. Gleitman, I990), and extended to domains such as the acquisition of adjectives and prepositions (see Bloom, I996, for review).

A second, distinct, source of linguistic information about the meaning of a word is provided by the semantics of the rest of the sentence. Imagine hearing the novel word gloobs in the sentence 'I ate several gloobs for breakfast yesterday'. Syntactically, the use of this word within the noun phrase ('several gloobs') reveals that it is a plural count noun, which in turn entails that it refers to multiple individuals, not to a property, an event, or a spatial relationship, and so on. Constrained only by this syntactic information, gloob could refer to eggs, to planets, to smiles, or to opinions, all of which are possible referents of plural count nouns. Plainly, however, we can sensibly infer a lot more about the meaning of gloob. For instance, a gloob is likely to be something that one can eat, it is presumably something that one would WANT to eat, one could eat more than one, it is likely to be smaller than a bread box, and so on. It is the SEMANTIC information carried by the rest of the sentence that serves to radically constrain the possible interpretations of the new word, to inform the word learner that it is more likely to refer to eggs than to conferences.

Following Wynn ( I 992b), we propose that children's earliest knowledge of number word meaning - that such words refer to precise numerosities comes through attention to both syntactic and semantic linguistic cues, such as the words' ordering relative to other words in a sentence, the closed-class morphemes they co-occur with, and the count-mass status of the nouns they modify. The information provided by relative order within the noun phrase is best viewed as syntactic, but other cues - in particular, those provided by closed-class morphology - are better construed as semantic. ${ }^{1}$ Although sensitivity to these different linguistic cues brings children to their initial stage of number word acquisition (knowledge that number words pick out numerosities), perhaps complete prior the age of two-and-a-half, a full understanding of the meanings of number words is only acquired through a distinct procedure (which we return to in the general discussion) that occurs later in development.

What evidence would bear on this hypothesis? If it is correct, three things

[I] Many scholars have suggested that information expressed by closed-class items is more constrained semantically than that expressed through open-class words and general contextual information (e.g. Pinker, I989). For instance, determiners can provide information about properties such as the discreteness, numerosity, shape, gender, and animacy of the referents of the nouns they co-occur with, but not information about their colour, temperature, hostility, and edibility. This suggests that the use of closed-class morphology as a cue to word meaning may utilize a more constrained inference process than inferences based on open-class morphemes such as the verb "eat", and thus can be viewed as intermediate between use of syntactic cues such as phrase-structure geometry on the one hand, and inference on the basis of general sentence meaning on the other. 
must follow. First, such cues must exist. That is, there must be linguistic contexts - in particular, contexts defined through word order and/or the relative location of certain closed-class morphemes - that select for words referring to numerosities, and not adjectives that are predicates of individuals, like big, or quantifiers that are predicates of portions, like much. Second, these cues must be present in the input that children receive. Third, children must themselves appreciate the relationship between number words and these linguistic cues. It would support this third claim if, in children's own spontaneous speech, number words only appear in certain unique linguistic contexts, the same contexts that, by hypothesis, inform them that these are in fact number words.

Consider the question of whether linguistic cues that the number words refer to numerosities actually exist. Number words do appear in some of the same surface positions as adjectives and other, semantically distinct, quantifiers, e.g. the two dogs waited, the little dogs waited, the many dogs waited. But other linguistic properties of number words differentiate them from adjectives and these quantifiers. Four such properties are discussed below:

I. Number words can be used only with count nouns, not with mass nouns. With regard to the sorts of nouns they co-occur with, number words fall into the same category as certain quantifiers, such as $a$, another, and many. They are distinct from other quantifiers, such as much (which can only co-occur with mass nouns), and all (which can co-occur with both count nouns and mass nouns). They are also distinct from most adjectives, which can appear with both count nouns and mass nouns (though some, such as big and long, typically appear only with count nouns, because their meanings require that they co-occur with nouns referring to individual entities with spatial or temporal extent, which are typically denoted by count nouns).

In languages like English, the grammatical distinction between count and mass nouns corresponds to a conceptual distinction between words that refer to individuals versus words that refer to non-individuated entities, or stuff (see Jackendoff, r 99 I ; Bloom \& Kelemen, I995). Four-year-olds can use the count-mass distinction to determine whether a word refers to an object versus a substance (Brown, 1957) or to a single sound versus an undifferentiated noise (Bloom, 1994a), and even two-year-olds are sensitive to this contrast when learning names for substances (mass nouns) versus names for bounded individuals consisting of substances, like puddles (count nouns) (Soja, 1992).

This contrast between count and mass nouns shows up early in language development, and Gordon (I988) has found that some children show productive command of the distinction by the age of $\mathrm{I} ; 9$. For these reasons, it seems plausible that the continued co-occurrence of number words with count nouns informs children about their meanings, that their semantic role is restricted to the quantification of INDIVIDUALS. 
2. Number words cannot appear with modifiers. The majority of adjectives are modifiable: one can specify the extent to which an adjective applies in a given instance by preceding it with a modifier, such as very, too, somewhat, and so on (e.g. The horses are too tired, Your face is quite red). Similarly, some quantifiers can be modified (e.g. That's too much putty, She has very few tension headaches, He says so many ridiculous things). Number words, however, do not pick out properties or states that can vary: they pick out discrete, absolute properties. Hence, they cannot occur with modifiers (e.g. phrases such as the very five salamanders and the dogs were so three are unacceptable).

This linguistic property of number words might reveal to the child that they are 'absolute' properties, which do not admit of variation. Of the four linguistic cues discussed here, this is the least syntactic. The information is carried by the meanings of modifiers. If children know what these words mean, they can infer upon hearing, for example, 'too much' or 'very nice' that much and nice refer to continuous properties. Note also that the potential to appear with modifiers delineates a semantic class that is at least partially orthogonal to syntax. For instance, some adjectives refer to numerical properties, such as even, odd, and prime. As with number words themselves, these cannot appear with modifiers. ${ }^{2}$

A learnability problem arises here, however, with regard to how children might use this cue in number word acquisition. Children are not given negative evidence; they are not explicitly told 'The phrase very two is ungrammatical', nor is there any reason to believe that all children produce errors like 'very two' and receive corrective feedback a significant proportion of the time that they do so (see Marcus, I 993). How then could a child know that number words CANNOT appear with modifiers - as opposed to inferring that he or she simply hasn't yet HEARD them appear with modifiers?

One possibility involves what is sometimes called 'indirect negative evidence' (Chomsky, I98I ; for discussion, see Pinker, I989; Valian, I990). If children hear a word frequently enough, but never in a certain context, they might infer it cannot be so used in that context. This may explain how children come to know that number words are restricted to count nouns. Given the fairly high frequency of mass noun usage, it is reasonable to infer from the fact that number words are never used with them that they CANNOT

[2] It is worth noting, however, that the examples with number words do sound wonse than those with adjectives - compare the very even number (awkward) with the very five dogs (completely unacceptable). This could be due to a purely syntactic difference between number words and adjectives. Alternatively, this continuum of modifiability may also be due to the semantics of the words themselves: some words, such as the number words and some quantifiers, are virtually impossible to think of as referring to properties on a continuum. In contrast, the types of discrete states denoted by adjectives allow for more cognitive flexibility, and hence we are able to sensibly interpret sentences like 'she's somewhat pregnant' or ' 3 is more prime than $23 \mathrm{II}$ '. 
be used with them. But this inference is less plausible with regard to modifiers, given their relatively low frequency. It might not be reasonable for a child to infer that just because a given number word has not yet been used with a modifier, that it cannot be so used.

This motivates another possibility. Children might start off with the assumption that all predicates cannot be used with modifiers, that they denote absolute properties. Under this account, only upon hearing a word used with a modifier will a child infer that it could refer to a continuous property. In other words, the child's null hypothesis, in the absence of any syntactic and semantic evidence to the contrary, is that all predicates are like number words in that they cannot be modified. If so, then the presence or absence of modifiers can serve as a cue to word meaning.

3. Number words precede adjectives within the noun phrase:they cannot follow them. In this respect, number words are similar to all other quantifiers. Just as two big dogs is acceptable and big two dogs is not, many big dogs is acceptable and big many dogs is not. Adjectives, however, can follow other adjectives (e.g. the brown spotted dogs), although there are certain ordering restrictions between different adjectives, based on their semantic properties (see Bever, I970).

Some semantic implications follow from this. Modifiers within the English noun phrase are analysed in a linear order, so that each modifier is a predicate of everything that follows. Thus the first adjective in big brown dogs modifies brown dogs, and the second modifies dogs. In this regard, the fact that number words (and other quantifiers) must precede adjectives within the noun phrase is informative as to their semantic nature. Adjectives describe properties of individuals; number words (and quantifiers) describe properties of sets of individuals. In a situation in which there are three dogs which are brown, it is the SET of (brown) dogs which is three, not each individual dog, and it is the dogs that are brown, not the set. Thus three brown dogs is acceptable and brown three dogs is not. ${ }^{3}$

4. Number words can occur in the partitive construction. The partitive construction in English is of the form __ of the Xs (Jackendoff, I977), and has

[3] For at least some dialects of English, there are exceptions to the generalization that number words can never precede adjectives within the Noun Phrase; these include phrases such as a wonderful three weeks and the gruelling ten miles we walked (Jackendoff, I 977). Why are these better than phrases such as the brown three dogs? The answer may lie in the fact that plural count nouns that denote measures of time or space can be construed as establishing reference to a single individual. Thus two weeks can denote a single continuous period of time of a certain duration - a temporal individual (and thus one can say a wonderful three weeks in Thailand is just what we need, using singular noun and verb agreement). In this regard, it is different from a phrase such as three dogs that almost always refers to a set of three distinct individuals. If three weeks can denote a single individual more readily than three dogs, then it follows that a wonderful three weeks should be more acceptable than the brown three dogs. 
the semantic role of expressing quantification, in which the first element 'extracts' either some group of individuals from the reference set denoted by the noun (some of the boys) or some portion of the non-individuated entity that is denoted (most of the water). Most quantifiers can appear in this context, and all number words can (two of the boys, etc.). Adjectives, as they lack the semantic capacity to quantify, cannot appear in the partitive construction.

Thus, we posit that the four linguistic cues above can tell children that number words refer to absolute quantities of discrete individuals - precisely the level of knowledge children were shown to possess in Wynn (1992b).

While there has been extensive research focusing on the social and pragmatic conditions under which adults use number words in their speech to children (e.g. Durkin, Shire, Reim, Crowther \& Rutter, I986; Saxe, Guberman \& Gearhart, I 987), there has yet been no systematic study of the syntax of number words in adults' speech to children and in children's own speech. To test the hypothesis that these four linguistic cues pick out number words in both the input to children and in children's own speech, we carried out analyses of transcripts of the spontaneous speech of children and their parents, from CHILdEs (MacWhinney \& Snow, I990).

\section{METHOD}

\section{Subjects}

Our choice of subjects was governed by the following considerations: ( I) The samples from the children had to be prior to the age of about three, so as to capture the developmental period identified by Wynn (I992b). (2) The samples should begin at a very early age, preferably prior to the age of two, so as to capture the onset of number word understanding. (3) There should be an adult caretaker in the samples, so we could explore the question of whether these linguistic cues are in the input. (4) There should be sufficient speech samples to permit within-child statistical analyses. (5) Ideally, the different children and adults should be from data samples of different scholars, so as to give us a heterogeneous sample.

We chose three subjects who met these criteria: Eve (from Brown, r 973), Peter (from L. Bloom, I970), and Naomi (from Sachs, I983). Information about the subjects is shown in Table $\mathrm{I}$.

тав LE I. Subjects tested from the CHILDES system

\begin{tabular}{lll}
\hline Child & Files & Age range \\
\hline Eve & I-20 & I $; 6$ to $2 ; 3$ \\
Peter & I-20 & I $; 9$ to $3 ;$ I \\
Naomi & I-80 & I $; 2$ to $2 ;$ I I \\
\hline
\end{tabular}


Procedure

We searched for number words, adjectives, and quantifiers, as shown in Table 2. We used the number words from two to ten. (Note that our

TABLE 2. Words searched for

\begin{tabular}{lll}
\hline Adjectives & Quantifiers & Number words \\
\hline hot & all & two \\
cold & another & three \\
new & any & four \\
old & both & five \\
green & few & six \\
yellow & many & seven \\
red & more & eight \\
blue & most & nine \\
good & much & ten \\
bad & only & \\
big & several & \\
little & some & \\
\end{tabular}

hypothesis is not intended to apply to one, since there is no evidence that children go through a stage in which they do not know the precise numerosity that it corresponds to.) The adjectives chosen are highly frequent in the speech of children and adults, as found in previous analyses by Nelson ( I 976) and Valian ( I986). The quantifiers were chosen so as to represent a large sample of different semantic types: we will discuss these types below.

All utterances containing these words in the speech of the children and their mothers were searched for using the CLAN program of the CHILDES system (MacWhinney \& Snow, I990). Verbatim repetitions were excluded from the analyses, as were those utterances that were unclassifiable for any reason. Each of the utterances was independently coded by the authors into one of $7 \mathrm{I}$ categories; coding disagreements were resolved by discussion. (Most of the 7 I category distinctions are irrelevant to the analyses below and were done for the purpose of further research using this database.)

RESULTS

Analyses I: Are number words used only with count nouns?

This first analysis tested the hypothesis that number words would only be used with count nouns, both in the input that children receive and in their own productive speech. In this regard, they should behave the same as the NON-MASS QUANTIFIERS few, many, both, several, and another, but behave differently from those quantifiers that can appear with mass nouns - the MASSABLE QUANTIFIERS more, some, most, only, all, any, and much (the only quantifier examined that can appear solely with mass nouns). We also 

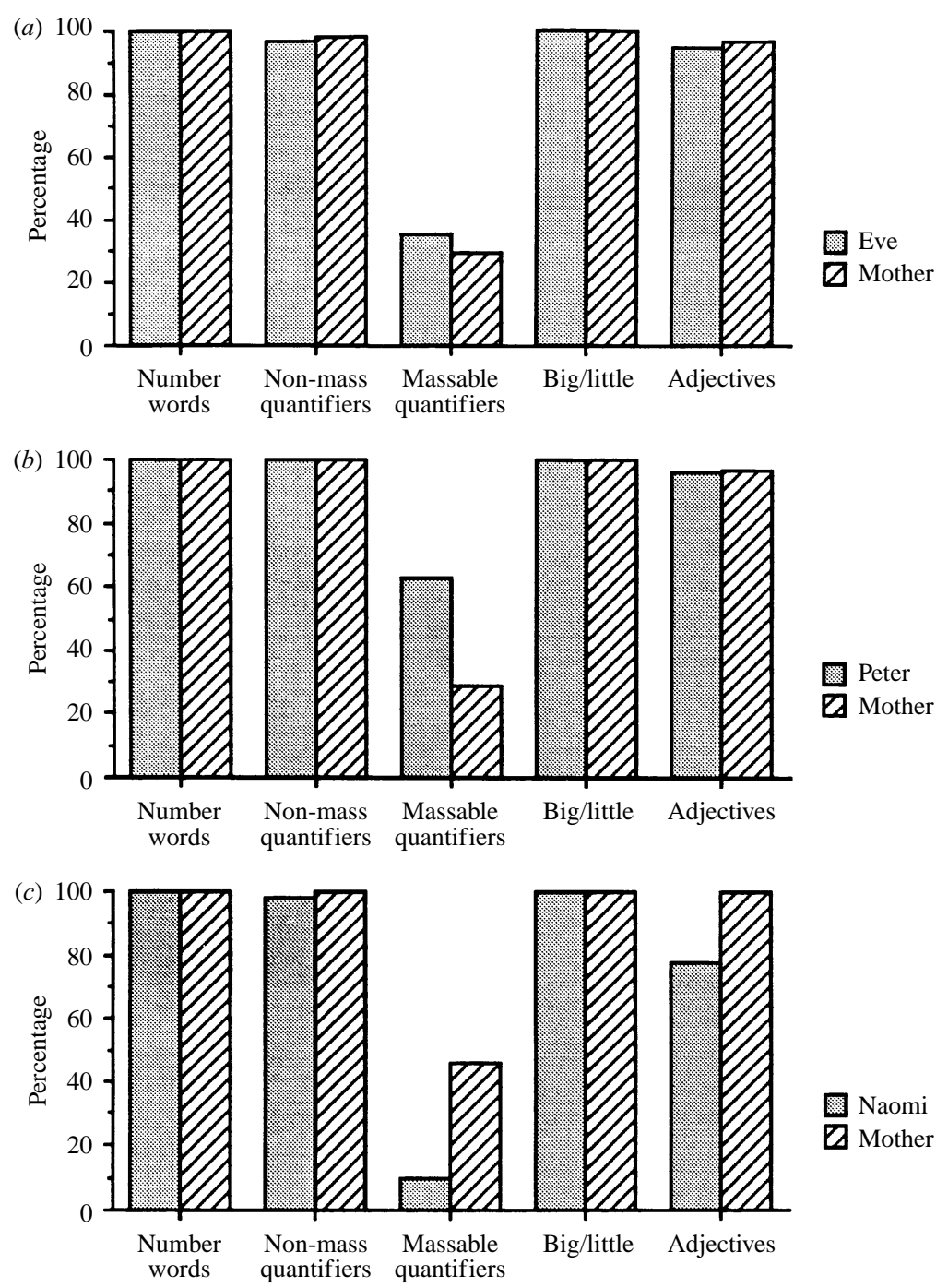

Fig. I. Percentage of utterances in which target words preceded a count noun, out of total number of utterances in which target words preceded a count or mass noun. Target word groups were: number words (two through ten), non-mass quantifiers (another, both, few, many, several), massable quantifiers (all, any, more, most, much, only, some), the adjectives big and little (which cannot occur with mass nouns), and the other adjectives.

predicted that number words would behave differently from all of the adjectives except for big and little, which, due to their semantics, almost always appear with count nouns.

The results are summarized in Fig. I above, where proportions are $52 \mathrm{I}$ 
calculated by determining the proportion of utterances in which number words co-occurred with count nouns from the total usage of number words that co-occurred with count noun and mass nouns (those nouns that have no clear preferential count/mass reading, like cake, were excluded from this analysis.)

We can consider each child in turn. Eve and Eve's mother always used number words with count nouns (Eve: 6o/6o tokens; Eve's mother: 43/43 tokens), and almost always used non-mass quantifiers like another with count nouns (Eve: I29/I33; Eve's mother: 95/97). In contrast, the massable quantifiers such as all and some, which have no such semantic selection requirement, were used only about one-third of the time with count nouns (Eve: 88/255; Eve's mother: 63/219). As predicted, the adjectives big and little were always used with count nouns (Eve: 98/98; Eve's mother: I०8/ı 8), but, contrary to our prediction, the other adjectives were also predominately used in this manner as well (Eve: 86/9I; Eve's mother: I I I / I I 5).

The results for Peter were virtually identical. Peter and Peter's mother always used number words with count nouns (Peter: I60/I60; Peter's mother: I5/I5), and always used non-mass quantifiers with count nouns (Peter: 209/209; Peter's mother: i6/ı6). But massable quantifiers were only used about one-half to one-quarter of the time with count nouns (Peter: 260/4I2; Peter's mother: 25/85). The adjectives big and little were always used with count nouns (Peter: 209/209; Peter's mother: 29/29), but, as with Eve, the other adjectives were also typically used in this manner as well (Peter: I37/I 43; Peter's mother: 33/34).

Finally, Naomi and Naomi's mother always used number words with count nouns (Naomi : 2 I/2 I ; Naomi's mother: I3/I3), virtually always used non-mass quantifiers with count nouns (Naomi: 62/63; Naomi's mother: $52 / 52)$, and used count nouns much less frequently with the massable quantifiers (Naomi: I I/I I I ; Naomi's mother: 8I/I76). The adjectives big and little were always used with count nouns (Naomi: 22/22; Naomi's mother : 64/64) and the other adjectives were used predominantly with count nouns as well (Naomi: 45/58; Naomi's mother: 53/53).

The main finding from this analysis, then, is that children and their parents only use number words with count nouns. In this regard, they use them in an identical fashion to quantifiers such as another and many, but differently from quantifiers such as all and much. (Chi-square analyses were done for each subject comparing the proportion of count nouns used with number words with the proportion of count nouns used with the massable quantifiers; all contrasts were highly significant: all $\chi^{2}>$ I 4 II I I ), all $p<$ ०.०० I.) On the other hand, there is no evidence that children semantically distinguish number words from adjectives or that such a distinction is present in the input that they receive. Note that this does not mean that 
children or adults use adjectives INCORRECTLY, but only that they do not use them in a manner that distinguishes them from number words with regard to their usage with count-mass syntax.

In sum, count nouns, but never mass nouns, co-occurred with number words, a pattern of usage shared by some but not all other quantifiers. This provides a cue to the meaning of number words - that they apply over INDIVIDUALs. There is no compelling information available from this source, however, to distinguish number words from adjectives.

\section{Analysis $2:$ Do number words appear with modifiers?}

Given that number words refer to discrete properties, they should not cooccur with modifiers like too and very. In this regard, they should fall into the same class as the non-modifiable quantifiers some, both, another, any, all, several, and only and should be distinct from the modifiable quantifiers much, many, most, and few and all of the adjectives, including big and little (more was excluded from this analysis because of its special nature; while it cannot be modified by typical modifiers, such as very, it can be modified by other quantifiers, such as some and much.

The results are shown in Fig. 2; proportions are calculated out of the total number of utterances in each word category, excluding unclassifiable utterances and counting contexts. Neither Eve nor her mother ever used modifiers with number words (Eve: o/69; Eve's mother: o/64) or nonmodifiable quantifiers (Eve: o/408; Eve's mother: o/4r9). This is not because modifiers were never used. In fact, they were used with modifiable quantifiers over half of the time (Eve: 4/7; Eve's mother: 19/32), and with adjectives, though less frequently (Eve: i I /27 I ; Eve's mother: 50/363). The difference between the proportion of number words used with modifiers and the proportion of modifiable quantifiers used with modifiers was highly significant for both Eve and her mother (Eve: $\chi^{2}=4 \mathrm{I} \cdot 62$ (I); Eve's mother: $\chi^{2}=47.38(\mathrm{I})$, both $\left.p<0.00 \mathrm{I}\right)$. The contrast between the proportion of modified number words and the proportion of modified adjectives was significant for Eve's mother $\left(\chi^{2}=9^{\circ} 99\right.$ ( I ), $\left.p<0.005\right)$, but not for Eve.

Peter and his mother showed the same pattern. Modifiers were never used with number words (Peter: ०/2I7; Peter's mother: $/ / 22$ ) or with nonmodifiable quantifiers (Peter: o/787; Peter's mother: o/ı82). They were used over half of the time with modifiable quantifiers (Peter: 16/20; Peter's mother: 8/14) and also used, less frequently, with adjectives (Peter: 57/5 I 7; Peter's mother: 22/I I4). This difference between the use of modifiers with number words and their use with modifiable quantifiers was significant for both Peter and his mother (Peter: $\chi^{2}=\mathrm{I} 86 \cdot \mathrm{I} 7$ (I); Peter's mother: $\chi^{2}=\mathrm{I} 6 \cdot \mathrm{I} 6$ ( I), both $p<0.00 \mathrm{I})$ and so was the difference between the use of modifiers with number words and their use with adjectives (Peter: $\chi^{2}=25^{\circ} 93$ (I); Peter's mother: $\chi^{2}=5.07(\mathrm{I})$, both $\left.p<0.05\right)$. 

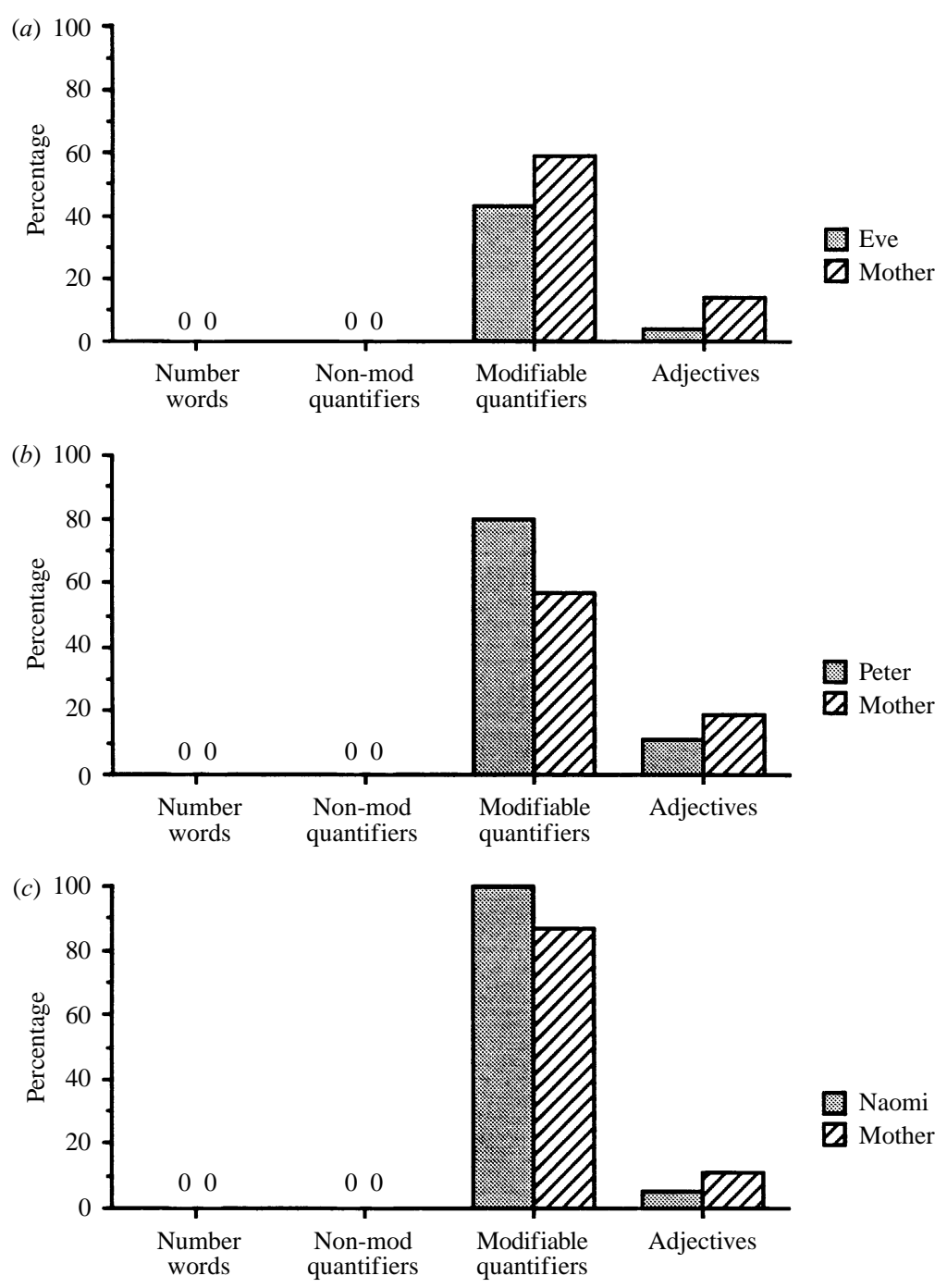

Fig. 2. Percentage of utterances in which target words were preceded by a modifier. Target word groups were: number words, non-modifiable quantifiers (all, another, any, both, only, several, some), modifiable quantifiers (few, many, most, much), and adjectives.

For Naomi as well, modifiers were never used with number words (Naomi: o/26; Naomi's mother: $0 / 22$ ) or with non-modifiable quantifiers (Naomi: o/ I 47 ; Naomi's mother: o/295). They were frequently used with modifiable quantifiers (Naomi: I/ I N Naomi's mother: 4I/47) and, less frequently, with adjectives (Naomi: 9/200; Naomi's mother: 26/233). The contrast between 
number words and modifiable quantifiers was significant for both Naomi and her mother (Naomi: $p=0 \cdot 04$, Fisher's exact test; Naomi's mother: $\chi^{2}=$ 47.29 ( I), $p<0.000 \mathrm{I}$ ). We should be cautious in interpreting the comparison for Naomi, however, since she only used one modifiable quantifier in the total sample (much). There was no significant difference between the extent of number word modification and the extent of adjective modification.

The results from this second analysis are clear. None of the children or their mothers used modifiers with number words or non-modifiable quantifiers such as another. But all of the children and their mothers used modifiers with modifiable quantifiers (such as much), and, to a lesser extent, with adjectives. This suggests that the semantic distinction between words denoting properties that fall on a continuum (e.g. size or magnitude) versus words denoting discrete properties (e.g. being of a certain numerosity) is present in the speech of parents and understood by children at an early age.

\section{Analysis 3: Are number words used only preceding adjectives?}

As discussed above, number words must occur before adjectives, not after them. This puts them into the same class as all other quantifiers and distinguishes them from adjectives, which can appear after one another (e.g. the nice big red house). For this analysis, we analysed only those target words that appeared in strings with adjectives, and calculated the proportion of these utterances in which the target word appeared before the adjective. The prediction was that number words and quantifiers should only appear in this context. (We also predicted that the adjectives we analysed should be equally distributed, but this is virtually a pre-ordained result, as most of the strings of more than one adjective that we found in our transcripts contained only those adjectives we were searching for. In such cases, one adjective gets counted as preceding an adjective, the other as following one-leading to a roughly $50 \%$ distribution.)

The results are shown in Figure 3 below. When number words were used with adjectives, the number word always preceded the adjective for Eve and her mother (Eve: I/ I ; Eve's mother: 6/6). Peter produced 9 utterances, one in which the number word followed the adjective ('too big two pockets'), thus getting $8 / 9$ correct (which suggests an understanding of the grammatical condition, as the odds of doing this well by chance would be $10 / 5 \mathrm{I} 2$, or $p=$ $0.02)$. Peter's mother produced a single number word-adjective sequence, in the correct order. Naomi never produced either an adjective-number word string or a number word-adjective string; Naomi's mother produced a single utterance of this type, with the number word preceding the adjective. For all the children and all the adults, quantifiers other than number words always preceded adjectives in adjective-quantifier strings (Eve: 6/6; 

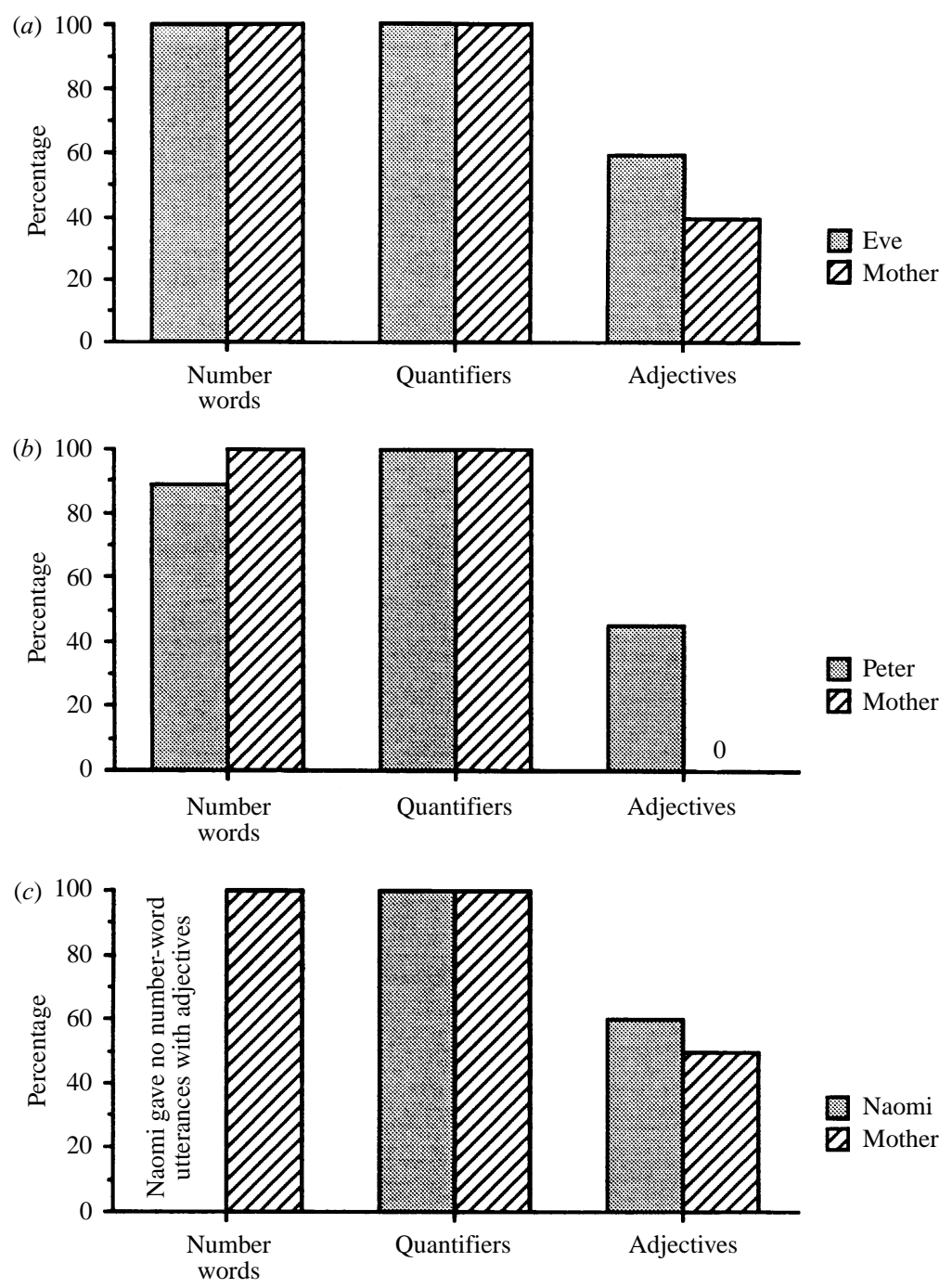

Fig. 3. Percentage of utterances in which target words preceded an adjective, out of total number of utterances in which target words either preceded or followed an adjective. Target word groups were: number words, quantifiers, and adjectives.

Eve's mother: 5/5; Peter: 6/6; Peter's mother: 3/3; Naomi: 3/3; Naomi's mother: 10/10).

These results are only significantly different from random variation for those subjects with a sample size of five utterances or more, and thus we can claim statistical confidence for only some of the subjects. Nevertheless, the 
consistently correct order is suggestive evidence for our hypothesis. The only error was produced by Peter (assuming that this was not a transcription error) and this was produced in the context of eight other correct orderings, suggesting that even he has the correct understanding.

\section{Analysis 4: Are number words and quantifiers - but not adjectives - used in the partitive construction?}

The usage of number words in the partitive construction, as with two of the $d o g s$, can serve as information that these words are predicates over sets of individuals, and not individuals themselves. In this regard, number words are identical to quantifiers (with the exception of only, which we excluded from this analysis) and different from all of the adjectives.

The results are shown in Figure 4 below. Partitive usages were relatively rare. Eve used partitives six times, all with quantifiers $(6 / 564)$, but never with number words. Eve's mother used them i 5 times, with both number words (3/64) and quantifiers (12/572). Thus while Eve herself displayed no knowledge that number words can appear in a partitive construction, the linguistic cues to that effect were present in her input. Peter used partitives 20 times, with both number words (2/217) and quantifiers (18/1 I 47), and his mother used them 14 times, also with number words $(3 / 22)$ and quantifiers (I I/229). As with the third analysis above, the data from Naomi are less revealing: she used the partitive construction only once (with a quantifier; I/269). Her mother used this construction 20 times, once with a number word (I/22) and the other times with quantifiers (19/407). The partitive construction was never used with adjectives, by any of our subjects.

The frequency of usage described above is sufficiently low that none of these contrasts is statistically significant, but if children can use the presence of a word within the partitive construction as a cue that it is a quantifier, the data are available for all of the children we studied.

The results from these analyses can be summarized as follows:

- Each of the three children and their mothers used number words only with count nouns, never with mass nouns. In this regard, they treated number words identically to quantifiers such as another, but differently from quantifiers such as all. They did not show a strong distinction between number words and adjectives, however, as adjectives were also predominantly used with count nouns.

- None of the three children or their mothers used modifiers with number words or with non-modifiable quantifiers such as another. Modifiers were used with modifiable quantifiers such as many, as well as with adjectives.

- When number words or other quantifiers appeared with adjectives in the surface string in the speech of the children and their mothers, these 

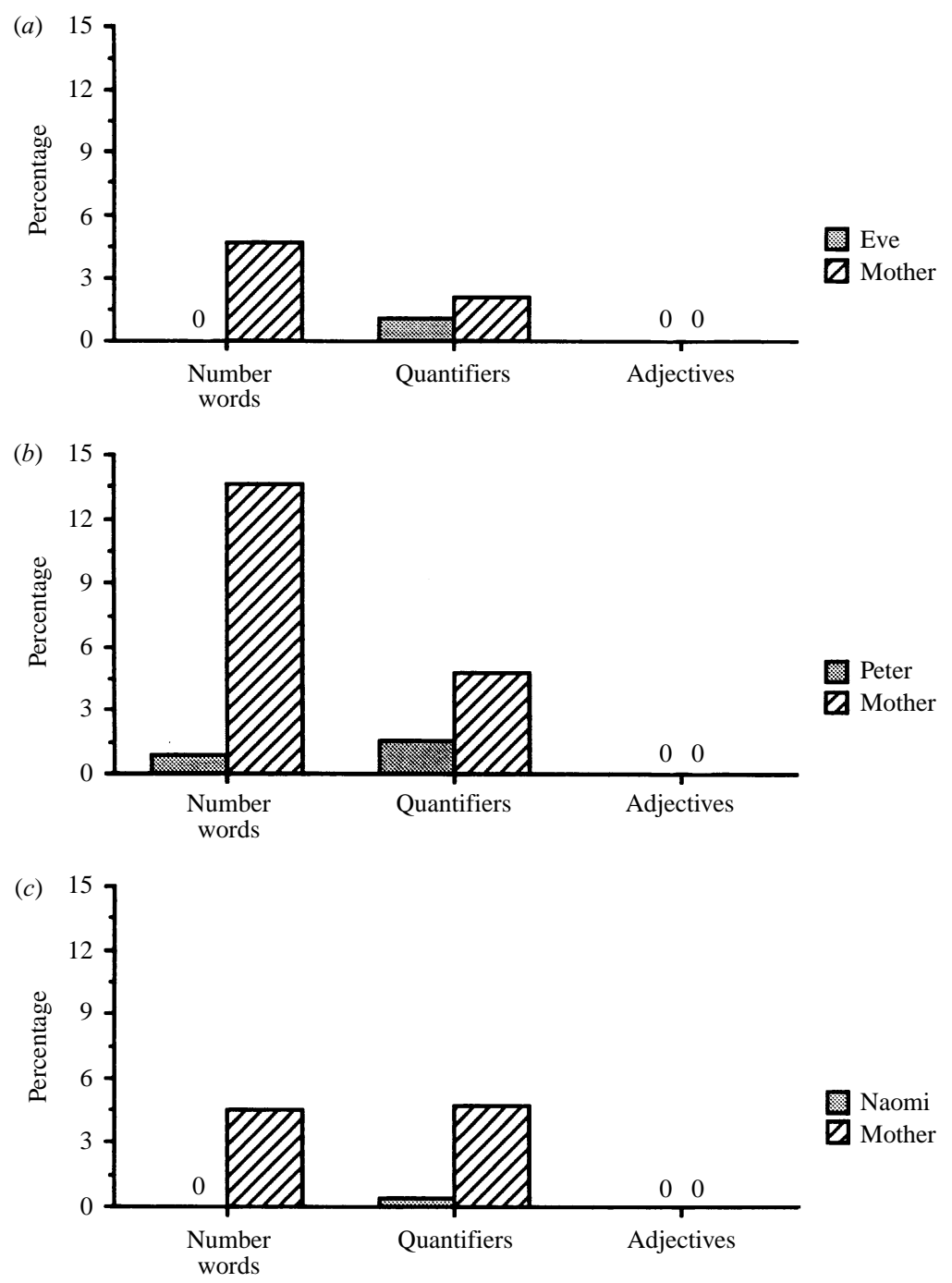

Fig. 4. Percentage of utterances in which target words occurred in a partitive construction. Target word groups were: number words, quantifiers, and adjectives.

words, with a single exception by Peter, always preceded the adjectives, providing evidence for a distinction between quantifiers and adjectives.

- The partitive construction was rare, but it occurred only with number words and other quantifiers, never with adjectives. 
GENERAL DISCUSSION

The analyses above suggests that in both the input to children and in their own speech, there is linguistic evidence consistent with the facts that number words apply over individuals (Analysis I), that they denote discrete values that do not permit modification (Analysis 2), and that they are quantifiers of sets (Analyses 3 and 4 ). These aspects of number word meaning coincide with the level of understanding that Wynn ( $1992 b$ ) found in her analysis of children's early understanding of the meanings of number words.

These results should be treated with caution, given the small and fairly homogeneous sample. Furthermore, these analyses do not show a causal relationship between linguistic cues and children's knowledge, only that the requisite linguistic cues to number word meaning exist in the input to children, and that children have some understanding of the nature of these cues. It is conceivable that children have learned that the number words refer to specific numerosities (but not their precise meanings) through some other, non-linguistic, learning mechanism, and thus that the linguistic information present in adult speech and understood by children plays no role in the learning process. A more direct test of our hypothesis would involve experimental manipulation, along the same lines as similar research on the role of linguistic cues in the acquisition of nouns and verbs. One could expose two-year-olds to novel words presented in the linguistic contexts explored here to see if this leads them to interpret the words as referring to specific numerosities.

A different way to explore the potential causal relationship between linguistic cues and the gradual acquisition of number word meanings would be by comparing the course of number word acquisition across different languages. For instance, children acquiring a language such as Straits Salish, in which there are no syntactic or morphological differences between number words and adjectives (Jelineke \& Demers, I994) should show a different pattern of emerging knowledge from that found in children acquiring English. In the extreme, children learning a language that does not linguistically distinguish number words from other predicates IN ANY REGARD must learn the meanings of number words exclusively through ostension and the counting system of their culture (assuming that there is one), and would not go through an initial stage in which they distinguish them from other predicates, such as big and many, but do not know their precise meaning. Less dramatic cross-linguistic differences, such as that between English and Japanese (which does not draw a morphological distinction between count nouns and mass nouns) should also have developmental ramifications.

The potential for cross-linguistic differences underscores an important aspect of the proposal made here, which is that the child's use of linguistic cues to infer number word meanings is assumed to occur on an 'op- 
portunistic' basis. That is, children come to have a tacit understanding, as a result of the normal process of syntactic and semantic development, that certain linguistic contexts correspond to certain classes of word meaning, and this knowledge facilitates the learning of words. This is quite different from the claim that humans have evolved an innate mechanism specifically devoted to exploiting linguistic cues in order to learn number words. We find such a claim implausible. Number words are likely to have been a relatively recent cultural advance, and some societies appear to get along with very few words denoting specific numerosities, sometimes only with the equivalents of one and two (Gordon, I 993).

Furthermore, a specialized mechanism for number word learning is not necessary. For instance, children know the relationship between the countmass status of nouns and the quantifiers they occur with as the result of word learning in general. Once children understand the syntax and semantics of water, they should be able to infer that a quantifier that interacts with water to establish reference to non-individual stuff cannot be a number word. Similarly, once children know what too and very mean, they can infer that quantifiers that occur with these modifiers must admit of variation along a continuum. What children need in order to achieve this first stage of number word understanding, then, is some general knowledge of the syntax and semantics of the language they are exposed to, not any learning mechanisms special to number.

How do children come to learn the number words' full meanings? From Wynn ( I 990, I 992b), we know that children first learn the precise meaning of one, then two, then three, and that this is a relatively prolonged process, suggesting that children are acquiring these words by focusing on the contexts in which they are used. Since humans have the capacity to apprehend the numerosity of small sets of items without conscious counting - an ability called 'subitizing' - this could be guiding children's acquisition of these first number words, assuming that there exists an antecedent mechanism that analyses the arrays to which these words apply into discrete individuals (Spelke, I 994).

After children learn three, which is probably the largest subitizable numerosity, there appears to be an explosion in their number word knowledge - they then acquire the precise meanings of four, five, six, and probably larger number words within their counting range (six was the largest word tested) all at once (Wynn, I992b). This occurs at precisely the same time that they come to understand how the counting system determines number (Wynn, I $992 b$ ), which suggests that an understanding of the counting system is necessary for acquiring the precise meanings of larger number words, as proposed by Gelman \& Gallistel ( I 978). More generally, this final aspect of number word learning appears to be the result of children hooking up their innate numerical understanding with the generative counting system, which 
might then give children the capacity to understand a potential infinity of number words (for discussion, see Bloom, 1994b).

How does this pattern of acquisition of the meanings of the number words relate to word learning in other domains? The proposal here motivates a more general perspective on the role of syntax in word learning and thereby addresses a central debate within the empirical and theoretical literature. On the one hand, scholars such as Gleitman (I 990) have stressed that syntactic cues play a significant role in word learning, and there are many empirical demonstrations that these cues serve to narrow down children's interpretation of the meaning of a novel word and to focus them on the appropriate ontological class. On the other hand - as stressed by Pinker (1989, i994)most aspects of word meaning are NOT reflected in the syntax, and thus no matter how rich the mappings children possess, they cannot solve the problem of word learning.

This tension motivates a hybrid theory, in which children first use linguistic cues to determine the broad semantic class of a novel word (kind of individual, kind of portion, quantifier, etc.) and then use other nonsyntactic information to determine the word's specific meaning. Number words are interesting because of the clear temporal demarcation between these two processes - the linguistic cues apply about a year before children manage to work out the words' specific meanings. But this hybrid account might nonetheless apply to the learning of nouns and verbs as well; it is just that the processes might occur so rapidly that their distinctness is impossible to observe.

Such a conclusion brings us back to a claim made at the beginning of this article. We argued that number words are different from words such as object names, in that they refer to properties of sets, not to individuals. The argument above, however, hints at a deeper commonalty across different classes of words. It suggests that the child's initial grasp of many word meanings is established by attending to their roles within sentences and inferring the broad semantic classes that they belong to. The rest of word learning - learning that cat refers to cats and not to dogs, that running refers to running and not to dancing, that two refers to two and not to twenty - is done through a distinct inferential process. In some important ways, then, perhaps number words are not so special after all.

\section{REFERENCES}

Bever, T. G. (1970). The cognitive basis for linguistic structures. In J. R. Hayes (ed.), Cognition and the development of language. New York, NY: Wiley.

Bloom, L. (1973). One word at a time: the use of single word utterances before syntax. The Hague: Mouton.

Bloom, P. (1 994a). Semantic competence as an explanation for some transitions in language development. In Y. Levy (ed.) Other children, other languages: issues in the theory of language acquisition. Hillsdale, $\mathrm{NJ}$ : Erlbaum. 


\section{BLOOM \& WYNN}

Bloom, P. ( $1994 b$ ). Generativity within language and other cognitive domains. Cognition 51 I $77-89$.

Bloom, P. ( r 996). Controversies in language acquisition: word learning and the part of speech. In R. Gelman \& T. Au (eds), Handbook of perceptual and cognitive development. New York: Academic Press.

Bloom, P. \& Kelemen, D. (I995). Syntactic cues in the acquisition of collective nouns. Cognition 56, I-30.

Brown, R. (1957). Linguistic determinism and the part of speech. Fournal of Abnormal and Social Psychology, 55, I-5.

Brown, R. (1973). A first language. Cambridge, MA: Harvard University Press.

Chomsky, N. (I98I). Lectures on government and binding. Dordrecht: Foris.

Clark, E. V. ( I 988). The principle of contrast: a constraint on language acquisition. In B. MacWhinney (ed.), Mechanisms of language acquisition. Hillsdale, NJ : Erlbaum.

Durkin, K., Shire, B., Reim, R., Crowther, R. D. \& Rutter, D. R. (I986). The social and linguistic context of early number word use. British Yournal of Developmental Psychology 4, 269-88.

Frege, G. ( I 893/ I 980). The foundations of arithmetic. Evanston, IL: Northwestern University Press.

Fuson, K. C. (1988). Children's counting and concepts of number. New York: Springer-Verlag.

Gelman, R. \& Gallistel, C. R. (I 978). The child's understanding of number. Cambridge, MA: Harvard University Press.

Gelman, R., Meck, E. \& Merkin, S. (r986). Young children's numerical competence. Cognitive Development $\mathbf{I}, \mathbf{I}-29$.

Gleitman, L. R. (I990). The structural sources of word meaning. Language Acquisition $\mathbf{r}$, 3-55.

Gordon, P. ( I 988). Count-mass category acquisition: distributional distinctions in children's speech. Fournal of Child Language $\mathbf{5}$, $109-28$.

Gordon, P. ( I 993). One-two-many systems in Amazonia: implications for number acquisition theory. Paper presented at the Society for Research in Child Development, New Orleans, March 25-28, I 993

Jackendoff, R. ( I 977). X-bar syntax : a study of phrase structure. Cambridge, MA : MIT Press.

Jackendoff, R. (I 99I). Parts and boundaries. Cognition 4I, 9-45.

Jelineke, E. \& Demers, R. A. ( I 994). Predicates and pronominal arguments in Straits Salish. Language 7o, 647-736.

Katz, N., Baker, E. \& Macnamara, J. T. (i 974). What's in a name? A study of how children learn common and proper names. Child Development 45, 469-73.

Macnamara, J. ( I982). Names for things: a study of human learning. Cambridge, MA: MIT Press.

MacWhinney, B. \& Snow, C. (r 990). The Child Language Data Exchange System: an update. Fournal of Child Language $\mathbf{1 7}, 457^{-72}$

Maddy, P. ( I 990). Realism in mathematics. New York: Oxford University Press.

Marcus, G. F. (I993). Negative evidence in language acquisition. Cognition 46, 53-85.

Markman, E. M. ( I989). Categorization and naming in children. Cambridge, MA: MIT Press.

Mill, J. S. ( I 843/ I 973). A system of logic ratiocinative and inductive, eighth edition. In J. M. Robson (ed.), Collected works of Fohn Stuart Mill (Vols 7, 8). University of Toronto Press.

Nelson, K. ( I 976). Some attributes of adjectives used by young children. Cognition 4, I-3 I.

Pinker, S. (1989). Learnability and cognition. Cambridge, MA: MIT Press.

Pinker, S. (I994). How could a child use verb syntax to learn verb semantics? Lingua 92, $377-410$.

Sachs, J. (I983). Talking about there and then: the emergence of displaced reference in parent-child discourse. In K. E. Nelson (ed.), Children's language (Vol. 4). Hillsdale, NJ : Erlbaum.

Saxe, J. B., Guberman, S. R. and Gearhart, M. (r987). Social processes in early number development. Monographs of the Society for Research in Child Development 52 (Serial No. 2 I6). 
Soja, N. N. (1992). Inferences about the meanings of nouns: the relationship between perception and syntax. Cognitive Development 7, 29-45.

Spelke, E. S. (I994). Initial knowledge: six suggestions. Cognition 5o, 43 I-45.

Starkey, P. \& Cooper, R. G., Jr. (1980). Perception of numbers by human infants. Science 2I0, I033-5.

Starkey, P., Spelke, E. S. \& Gelman, R. (I990). Numerical abstraction by human infants. Cognition 36, 97- 28 .

Valian, V. (1986). Syntactic categories in the speech of young children. Developmental Psychology 22, 562-79.

Valian, V. (I990). Null subjects: a problem for parameter-setting models of language acquisition. Cognition 35, 105-22.

Wynn, K. (1990). Children's understanding of counting. Cognition 36, I 55-93.

Wynn, K. (I $992 a$ ). Addition and subtraction by human infants. Nature 358, $749^{-} 5^{\circ}$.

Wynn, K. (1992b). Children's acquisition of the number words and the counting system. Cognitive Psychology 24, 220-5 I.

Wynn, K. (I 996). Infants' individuation and enumeration of actions. Psychological Science 7, I 64-9. 